\title{
1 ClinePlotR: Visualizing genomic clines and detecting outliers in $\mathbf{R}$
}

3 Bradley T. Martin ${ }^{*}, 1,2$ | Tyler K. Chafin ${ }^{1} \mid$ Marlis R. Douglas ${ }^{1} \mid$ Michael E. Douglas ${ }^{1}$

$5 \quad{ }^{1}$ Department of Biological Sciences, University of Arkansas, Fayetteville, AR 72701 USA

$6 \quad{ }^{2}$ Global Campus, University of Arkansas, Fayetteville, AR 72701 USA

8 E-mail: btm002@uark.edu (BTM); tkchafin@uark.edu (TKC); mrd1@uark.edu (MRD);

9 med1@uark.edu (MED)

11 Correspondence author. E-mail: btm002@uark.edu

13 ORCID

14 BTM: https://orcid.org/0000-0002-3014-4692

15 TKC: $\underline{\text { https://orcid.org/0000-0001-8687-5905 }}$

16 MRD: https://orcid.org/0000-0001-6234-3939

17 MED: https://orcid.org/0000-0001-9670-7825

18

19 Running Title: Visualizing genomic clines and outliers 


\section{Abstract}

23 Patterns of multi-locus differentiation (i.e., genomic clines) often extend broadly across hybrid

24 zones and their quantification can help diagnose how species boundaries are shaped by adaptive

25 processes, both intrinsic and extrinsic. In this sense, the transitioning of loci across admixed

26 individuals can be contrasted as a function of the genome-wide trend, in turn allowing an

27 expansion of clinal theory across a much wider array of biodiversity. However, computational

28 tools that serve to interpret and consequently visualize 'genomic clines' are limited. Here, we

29 introduce the CLINEPLOTR R-package for visualizing genomic clines and detecting outlier loci

30 using output generated by two popular software packages, BGC and INTROGRESS. CLINEPLOTR

31 bundles both input generation (i.e, filtering datasets and creating specialized file formats) and

32 output processing (e.g., MCMC thinning and burn-in) with functions that directly facilitate

33 interpretation and hypothesis testing. Tools are also provided for post-hoc analyses that interface

34 with external packages such as ENMEVAL and RIDEOGRAM. Our package increases the

35 reproducibility and accessibility of genomic cline methods, thus allowing an expanded user base

36 and promoting these methods as mechanisms to address diverse evolutionary questions in both

37 model and non-model organisms.

39 Keywords: hybrid zones, BGC, INTROGRESS, population genetics, bioinformatics, selection 


\section{Introduction}

Patterns of multi-locus differentiation, as distributed across admixture gradients, have

42 long provided a window into divergence and speciation (e.g., Barton, 1983; Gompert,

43 Mandeville, \& Buerkle, 2017). Accordingly, they have been used to map loci associated with

44 adaptation or reproductive isolation (Buerkle \& Lexer, 2008; Martin et al., 2020), and as

45 indicators of biotic responses to environmental change (Chafin, Douglas, Martin, \& Douglas,

46 2019). Rather than relating these to patterns in the landscape, contemporary approaches have

47 instead drawn conclusions based on genome-wide ancestries (Gompert \& Buerkle, 2009;

48 Fitzpatrick, 2013). The evolutionary processes that generate 'genomic clines' can be illuminated

49 even when constituent taxa do not segregate geographically, but rather patchily (Bierne,

50 Gagnaire, \& David, 2013), or as a hybrid mosaic (Chafin et al., 2019).

51 Several programs are available specifically to investigate genomic clines. Of these, BGC

52 (Gompert \& Buerkle, 2011, 2012) is the most robust to false positives and uses a Bayesian

53 approach that accounts for genotype uncertainty (Gompert, Lucas, et al., 2012) and

54 autocorrelation caused by physical linkage (Gompert, Parchman, \& Buerkle, 2012) in next-

55 generation sequencing datasets. Although a powerful tool for analyzing hybridization with

56 molecular data, it lacks user-friendly output. Researchers must either develop custom scripts or

57 build cumbersome, one-off pipelines, neither of which is parsimonious. A more direct approach

58 is clearly necessary.

Here, we present a comprehensive R-package, CLINEPLOTR, that promotes the genomic

60 cline methodology. The package includes functions that facilitate BGC input file generation and

61 output visualization and extend the plotting functionality from another genomic cline software

62 package, INTROGRESS (Gompert \& Buerkle, 2010). Locus-wise clinal patterns are visualized by 
63 accessing a suite of R-methods that interpret them as a function of the genome-wide average,

64 genomic position along chromosomes, and in relation to spatial and environmental parameters.

\section{Description}

\section{OVERALL PACKAGE WORKFLOW}

The CLINEPlotR R-package incorporates an introduction to available functions and can

69 be installed via provided instructions directly from the GitHub repository

70 (github.com/btmartin721/ClinePlotR). CLINEPLOTR includes three primary pipelines, a summary

71 of which can be visualized in Figure 1.

72 The workflow for our BGC pipeline includes functions to aggregate outputs from multiple

73 independent runs, thin MCMC samples, and plot log-likelihood and BGC parameter traces. From

74 these, ClinePlotR can both identify outlier loci using any of several user-defined options and

75 plot locus-wise ancestry probabilities $(\phi)$ as a function of the hybrid index (Figure 2). Finally,

76 users can examine the locus-wise relationship between cline center $(\alpha)$ and rate $(\beta)$, with

77 polygon hulls included to encapsulate 2D 'outlier space' for each parameter (Gauthier et al.,

78 2020).

CLINEPLOTR additionally includes accessory functions that allow an examination of

80 variation in clinal parameters across the genome. Although mapping loci to reference assemblies

81 is outside the scope of this package, an example of a workflow using MINIMAP2 (Li, 2018) is in

82 the documentation. If the user has access to physical SNP (single nucleotide polymorphism)

83 coordinates and a closely-related chromosome-level assembly, CLINEPLOTR can integrate these

84 data with the RIDEOGRAM package (Hao et al., 2020) to yield karyotype-style ideograms

85 annotated with heatmaps for both BGC cline parameters (Figure 3). 
Functions are also provided to facilitate an INTROGRESS workflow by generating input

87 data frames as well as accessories that embellish the plotting functions already present in

88 INTROGRESS. These accessory functions will visualize spatial patterns (e.g., latitude/ longitude)

89 and environmental variables that are inherent to genomic clines (Figure 4), to include helper

90 functions that invoke ecological niche models (MAXENT: Phillips, Anderson, \& Schapire, 2006)

91 as generated in the R-package ENMEVAL (Muscarella et al., 2014).

The primary purpose of CLINEPLOTR is to simplify the use of rather cumbersome

95 software designed to estimate genomic clines. To facilitate this task, accessory scripts that

96 prepare files for input into BGC and INTROGRESS are available in the GitHub repository, with a

97 few variants. For example, phylip2bgc.pl script converts a PHYLIP-formatted alignment

98 containing concatenated SNPs to the custom BGC input format. It can also subset populations

99 and/ or individuals from a larger alignment. A similar script, phylip2introgress.pl, does likewise

100 with INTROGRESS input. Because BGC can additionally consider linkage among loci as well as

101 genotype uncertainty, an input script (vcf2bgc.py) that employs the PYVCF Python library

102 (https://pyvcf.readthedocs.io/) is also provided as a means to format an IPYRAD (Eaton \&

103 Overcast, 2020) VCF file containing annotations for physical position and genotype read counts.

104 Finally, an additional script, nremover.pl, is provided to comprehensively filter a PHYLIP-

105 formatted SNP file. The program includes the capacity to filter by matrix occupancy per

106 individual and per SNP column, and by minor allele frequency. It will also remove non-biallelic

107 or monomorphic SNPs, and can randomly subsample large datasets. 
111 prefix_bgc_stat_param_replicate, where prefix is shared across all independent BGC replicates,

112 param is an individual output parameter (e.g., LnL), and replicate is an integer. Outputs from

113 any number of replicates can then be parsed, thinned, and combined via the combine_bgc_output

114 function in CLINEPLOTR. The combine_bgc_output function provides arguments for the number

115 of MCMC samples to be removed as burn-in, and for a sampling frequency with which to thin

116 samples. Following BGC run aggregation, the MCMC samples can be visually inspected for

117 mixing and convergence using a trace plotting function, plot_traces. Adjustments can then be

118 made to thinning or burn-in parameters by re-running the combine_bgc_output function or, if

119 necessary, by re-running BGC with altered parameters or increased MCMC length.

121 ancestry or exceptionally steep transitions relative to the genome-wide average. Here, we provide

122 the function get_bgc_outliers that offers two outlier detection methods [described in Gompert \&

123 Buerkle $(2011,2012)]$. Briefly, the first simply queries if the credibility intervals for the

124 posterior probability distribution of cline parameters $\alpha$ or $\beta$ (i.e., cline center and rate,

125 respectively) exclude the neutral expectation (i.e., $\alpha$ or $\beta=0$ ). If this interval excludes zero for

126 either parameter, a locus can be flagged as either an $\alpha$-outlier, $\beta$-outlier, or both.

The second method considers if per-locus parameter estimates are statistically unlikely,

128 given the distribution of values across all loci. This is accomplished by classifying outliers as

129 those for which posterior median $\alpha$ and $\beta$ estimates are not encapsulated by the $\left(\frac{n}{2}\right)$ and $\left(\frac{1-n}{2}\right)$

130 quantiles from a conditional $\alpha$ and $\beta$ prior distribution (Gaussian with a mean of zero), where $n$

131 represents a user-specified threshold (e.g., 95\%, 97.5\%). Users can choose whether to classify 
132 outliers using any combination of the above methods, but all require the zeta and gamma quantile

133 estimates from the BGC output.

134 We additionally track whether parameter values are significantly positive or negative.

135 This indicates either an increase $(\alpha>0)$ or decrease $(\alpha<0)$ in the probability of parental

136 population ancestry among hybrids for a given locus, or deviation in the rate of transition in

137 probabilities of locus-specific ancestries towards either very steep $(\beta>0)$ or wide $(\beta<0)$ shapes

138 (Gompert \& Buerkle, 2011).

140 VISUALIZATION OPTIONS

141 We attempted to tailor available visualizations in CLINEPLOTR towards common

142 applications of Bayesian genomic clines found in the literature, and we will continue to add

143 additional ones as need arises. Many applications seek to identify loci subject to various selective

144 processes (Parchman et al., 2013) by comparing how ancestries transition among loci with

145 respect to the genome wide average. To facilitate this, the phiPlot function computes $\phi_{\mathrm{ijn}}$, the

146 probability of parental population1 ancestry for each locus $(i)$ and individual $(n)$ within each

147 admixed population (j) [Eqn. 3 and 4; Gompert \& Buerkle (2011)]. It then produces a plot of $\phi$

148 (per locus) on the $\mathrm{y}$-axis against posterior estimates of hybrid index on the x-axis (sensu

149 Gompert et al., 2012), with a user-specified color scheme that designates statistical outliers

150 (Figure 2).

151 Other applications have specifically examined relationships among cline rate and center

152 parameters (Gauthier et al., 2020), and we also do so by implementing the alphaBetaPlot

153 function. A 2-D density contour plot of $\alpha$ and $\beta$ parameters is produced, with values for 
154 individual loci optionally mapped, and with the potential to calculate and plot polygon hulls that

155 encapsulate positive and negative outliers with respect to each parameter (Figure 2).

\section{EXTENDED FUNCTIONS AND HELPER SCRIPTS}

We also provide several additional functions (see Figure 1) that have considerable use cases, although some seemingly deviate from the 'core' BGC workflow. The first of several can

160 be used to map parameter values of BGC clines onto a chromosomal ideogram via the function

161 plot_outlier_ideogram (e.g., Figure 3), Here, BGC results are depicted for a case study examining

162 hybridization between Woodland (Terrapene carolina carolina) and Three-toed box turtles

163 (Terrapene mexicana triunguis) (Martin et al., 2020). However, some external user-steps are

164 required to use the function.

165 Briefly, we mapped the Terrapene ddRAD sequencing alignment against the available

166 Terrapene mexicana triunguis scaffold-level assembly (GenBank Accession:

167 GCA_002925995.2). Scaffold coordinates were then converted to chromosome coordinates by

168 mapping Terrapene scaffolds against the closely related chromosome-level Trachemys scripta

169 assembly [(Simison, Parham, Papenfuss, Lam, \& Henderson, 2020); GenBank accession:

170 GCA_013100865.1]. This was accomplished by employing MINIMAP2 (Li, 2018) and PAFSCAFF

171 (github.com/slimsuite/pafscaff). The output from get_bgc_outliers and PAFSCAFF, plus a GFF

172 file read/ parsed via the provided functions parseGFF and join_bgc_gff, were used to plot a

173 heatmap of BGC $\alpha$ - and $\beta$-values on an ideogram. Essentially, the ideogram plot (generated using

174 the RIDEOGRAM R-package) allows the chromosomal locations of each outlier to be visualized

175 (Figure 3). It also provides a distinction between transcriptomic SNPs falling within known 
176 genes versus loci from surrounding scaffolds. For additional details, a more in-depth tutorial is

177 provided at github.com/btmartin721/ClinePlotR.

178 Other extended functions include a wrapper to simplify running INTROGRESS

179 (runIntrogress), and a function that allows genomic clines (Figure 4A) and hybrid indices

180 (Figure 4B) from INTROGRESS to be correlated with spatial and environmental variables. To

181 access this functionality, one can run clinesXenvironment using the object returned from

182 runIntrogress and raster values extracted from each sample locality. Multiple rasters can be

183 included (e.g., the 19 BioClim layers; https://worldclim.org/), and users can run the included

184 ENMEVAL wrapper functions (runENMeval and summarize_ENMeval) to identify uninformative

185 layers that may subsequently be excluded from clinesXenvironment. These latter functions access

186 MAXENT using the ENMEVAL pipeline (Muscarella et al., 2014), whereby the most informative

187 raster layers are designated with the 'permutation importance' statistic.

Conclusions

Genomic clines are useful for assessing patterns of introgression in hybrid zones.

191 Unfortunately, parsing and plotting results from the available genomic cline software can be

192 difficult. Given that genomic clines have a variety of applications, to include conservation

193 genetics, evolutionary biology, and speciation research, it is clearly important that they be

194 accessible for use by researchers. Here, we present an R-package that greatly simplifies the

195 parsing of output from available genomic cline software, as well as the production of

196 publication-quality figures. Our R-functions are intended to be user-friendly, and to this end

197 employ a variety of parameters that can be altered by users to suit specific research needs.

198 Furthermore, CLINEPLOTR allows outlier SNPs to be visualized across the genome, while also 
199 distinguishing known genes and surrounding loci. In addition, the environmental and spatial

200 effects on genomic clines can be assayed. This extended functionality enhances the interpretation

201 of genomic clines and provides greater insight into those underlying processes that potentially

202 contribute to the observed patterns. Hopefully, future iterations of genomic cline software can act

203 to extend chromosomal and environmental associations, particularly as whole genome

204 sequencing becomes less expensive and more common.

206 Funding

207 No external funding was provided to the authors in support of this work.

209 Acknowledgements

210 We thank S.M. Mussmann and M.R. Bangs for contribution to the nremover.pl script. We also

211 thank staff of the Arkansas High Performance Compute Center (AHPCC) for access to

212 computational resources that were used to test this package on the University of Arkansas

213 Trestles cluster (funded through multiple National Science Foundation grants and the Arkansas

214 Economic Development Commission).

215

216 Authors' contributions

217 BTM and TKC developed the R-package and wrote the manuscript and all code. MRD and MED

218 were the study supervisors. All co-authors contributed to editing the manuscript.

220 Data accessibility 
221 ClinePlotR is available as a GitHub repository: https://github.com/btmartin721/ClinePlotR. We

222 also plan to submit to CRAN prior to publication. The data used herein will be available as an

223 example dataset in a Dryad Digital Repository [DOI]. During review, the data will also be

224 temporarily accessible from Box Drive

225 (https://uark.box.com/s/ei21v3unvxfxczbfnfflwhrk536bsd5h) 


\section{References}

227 Barton, N. H. (1983). Multilocus Clines. Evolution, 37(3), 454-471. doi:10.2307/2408260

228 Bierne, N., Gagnaire, P. A., \& David, P. (2013). The geography of introgression in a patchy

229 environment and the thorn in the side of ecological speciation. Current Zoology, 59(1), 72-

230 86. doi:https://doi.org/10.1093/czoolo/59.1.72

231 Buerkle, C. A., \& Lexer, C. (2008). Admixture as the basis for genetic mapping. Trends in

232 Ecology and Evolution, 23(12), 686-694. doi:10.1016/j.tree.2008.07.008

233 Chafin, T. K., Douglas, M. R., Martin, B. T., \& Douglas, M. E. (2019). Hybridization drives genetic erosion in sympatric desert fishes of western North America. Heredity, 123, 759773. doi:10.1038/s41437-019-0259-2

Eaton, D. A. R., \& Overcast, I. (2020). ipyrad: Interactive assembly and analysis of RADseq datasets. Bioinformatics, 36(8), 2592-2594. doi:10.1093/bioinformatics/btz966

238 Fitzpatrick, B. M. (2013). Alternative forms for genomic clines. Ecology and Evolution, 3(7), 1951-1966. doi:https://doi.org/10.1002/ece3.609

240 Gauthier, J., de Silva, D. L., Gompert, Z., Whibley, A., Houssin, C., Le Poul, Y., ... Elias, M.

241 (2020). Contrasting genomic and phenotypic outcomes of hybridization between pairs of mimetic butterfly taxa across a suture zone. Molecular Ecology, 29(7), 1328-1343.

244 Gompert, Z., \& Buerkle, C. A. (2009). A powerful regression-based method for admixture 245 mapping of isolation across the genome of hybrids. Molecular Ecology, 18(6), 1207-1224. doi:10.1111/j.1365-294X.2009.04098.x

247 Gompert, Z., \& Buerkle, C. A. (2010). INTROGRESS: a software package for mapping 
components of isolation in hybrids. Molecular Ecology Resources, 10(2), 378-384.

doi:10.1111/j.1755-0998.2009.02733.x

250

Gompert, Z., \& Buerkle, C. A. (2011). Bayesian estimation of genomic clines. Molecular Ecology, 20(10), 2111-2127. doi:10.1111/j.1365-294X.2011.05074.X

Gompert, Z., \& Buerkle, C. A. (2012). BGC: Software for Bayesian estimation of genomic clines. Molecular Ecology Resources, 12(6), 1168-1176. doi:10.1111/1755-0998.12009.x

Gompert, Z., Lucas, L. K., Nice, C. C., Fordyce, J. A., Forister, M. L., \& Buerkle, C. A. (2012). Genomic regions with a history of divergent selection affect fitness of hybrids between two butterfly species. Evolution, 66(7), 2167-2181. doi:10.1111/j.1558-5646.2012.01587.x

Gompert, Z., Mandeville, E. G., \& Buerkle, C. A. (2017). Analysis of population genomic data from hybrid zones. Annual Review of Ecology, Evolution, and Systematics, 48, 207-229. doi:10.1146/annurev-ecolsys-110316-022652

Gompert, Z., Parchman, T. L., \& Buerkle, C. A. (2012). Genomics of isolation in hybrids. Philosophical Transactions of the Royal Society B: Biological Sciences, 367(1587), 439_ 450. doi:10.1098/rstb.2011.0196

Hao, Z., Lv, D., Ge, Y., Shi, J., Weijers, D., Yu, G., \& Chen, J. (2020). RIdeogram: drawing SVG graphics to visualize and map genome-wide data on the ideograms. PeerJ Computer Science, 6, e251. doi:10.7717/peerj-cs.251

Li, H. (2018). Minimap2: pairwise alignment for nucleotide sequences. Bioinformatics, 34(18), 3094-3100. doi:10.1093/bioinformatics/bty191

Martin, B. T., Douglas, M. R., Chafin, T. K., Placyk, J. S., Birkhead, R. D., Phillips, C. A., \& Douglas, M. E. (2020). Contrasting signatures of introgression in North American box turtle 
271 Muscarella, R., Galante, P. J., Soley-Guardia, M., Boria, R. A., Kass, J. M., Uriarte, M., \&

272 Anderson, R. P. (2014). ENMeval: An R package for conducting spatially independent

273 evaluations and estimating optimal model complexity for Maxent ecological niche models .

$274 \quad$ Methods in Ecology and Evolution, 5(11), 1198-1205. doi:10.1111/2041-210x.12261

275 Parchman, T. L., Gompert, Z., Braun, M. J., Brumfield, R. T., McDonald, D. B., Uy, J. a C., ...

276 Buerkle, C. a. (2013). The genomic consequences of adaptive divergence and reproductive

277 isolation between species of manakins. Molecular Ecology, 22(12), 3304-3317.

$278 \quad$ doi: $10.1111 / \mathrm{mec} .12201$

279 Phillips, S. J., Anderson, R. P., \& Schapire, R. E. (2006). Maximum entropy modeling of species

280 geographic distributions. Ecological Modeling, 190(3), 231-259.

281 doi:10.1016/j.ecolmodel.2005.03.026

282 Simison, W., Parham, J., Papenfuss, T., Lam, A., \& Henderson, J. (2020). Annotated

283 chromosome-level reference genome of the red-eared slider turtle (Trachemys scripta

284 elegans). Genome Biology and Evolution, 12(4), 456-462. doi:10.1093/gbe/evaa063 

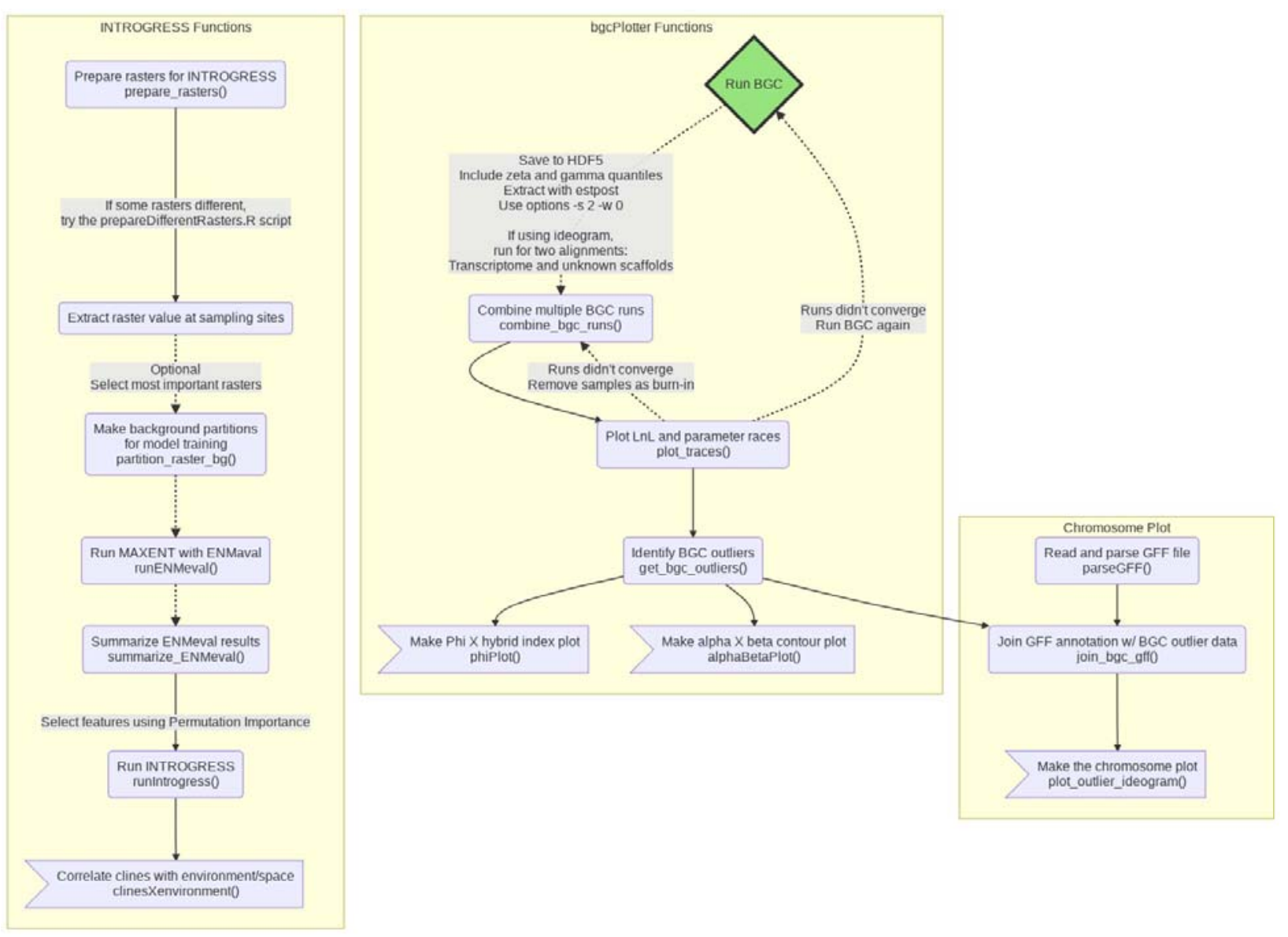

289 Figure 1: Simplified example workflow listing all available CLINEPLOTR functions. Yellow

290 boxes group inter-dependent functions working towards producing one or two particular plots

291 (terminal plotting steps depicted as flags). Connecting arrows indicate a pipeline where each step

292 is dependent on the returned R objects. The green 'Run BGC' diamond identifies BGC as an

293 external a priori step for the bgcPlotter and chromosome plot functions. The dotted lines indicate

294 optional steps. 


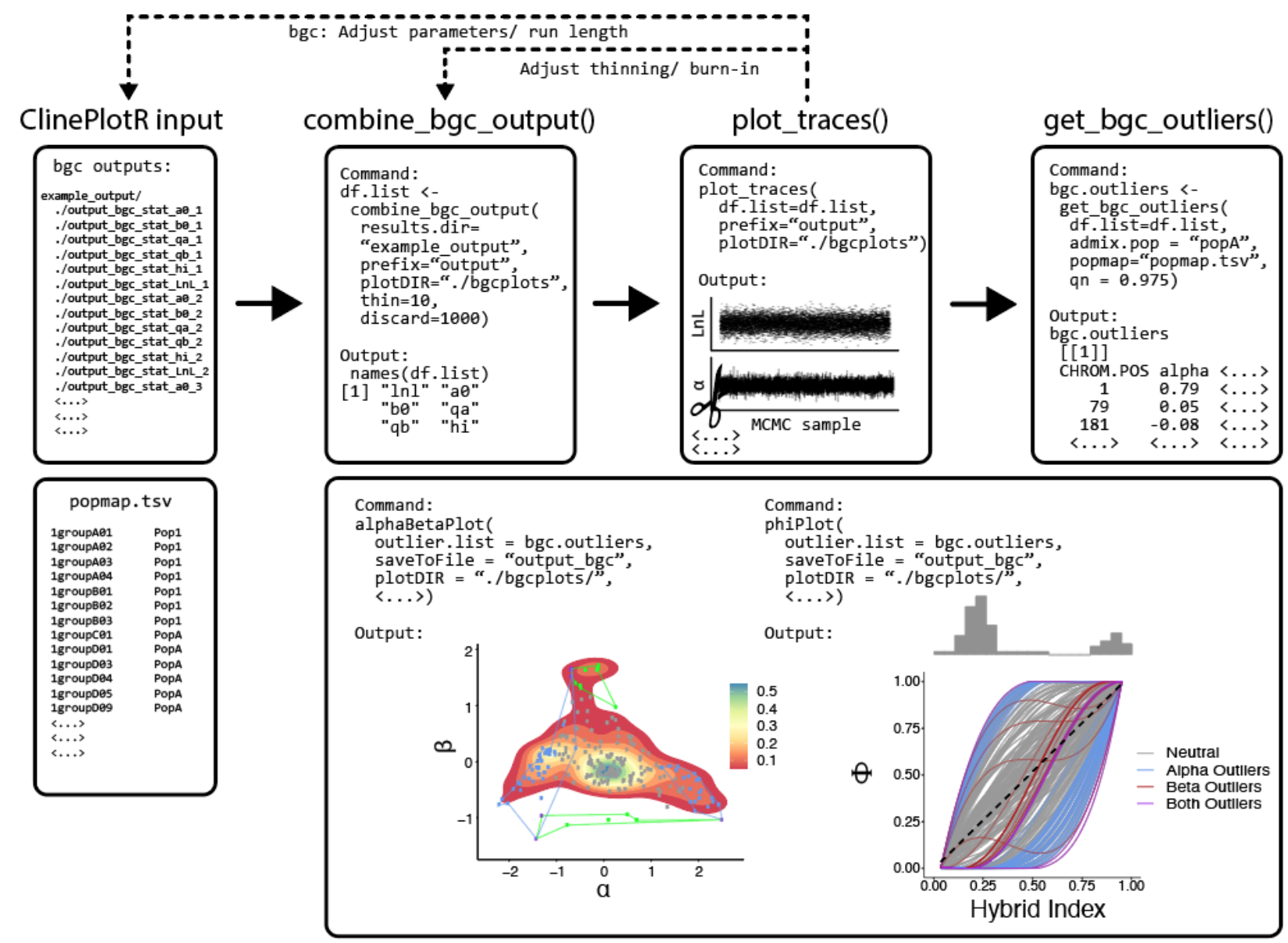

297 Figure 2. Example workflow for parsing Bayesian genomic cline (BGC) output, visualizing

298 MCMC traces, detecting outliers, and plotting results. The 'phiPlot' (right-side, lower right box)

299 shows hybrid indices (x-axis) and probability of parental population1 alleles (y-axis), plus a

300 histogram of hybrid indices in the admixed population. The 'alphaBetaPlot' (left-side, lower

301 right box) shows 2-D density of cline width/ rate representing the cline center (i.e., bias in SNP

302 ancestry; $\alpha$; $\mathrm{x}$-axis) and steepness of clines ( $\beta$; y-axis). Outliers are additionally encapsulated

303 using polygon hulls. 


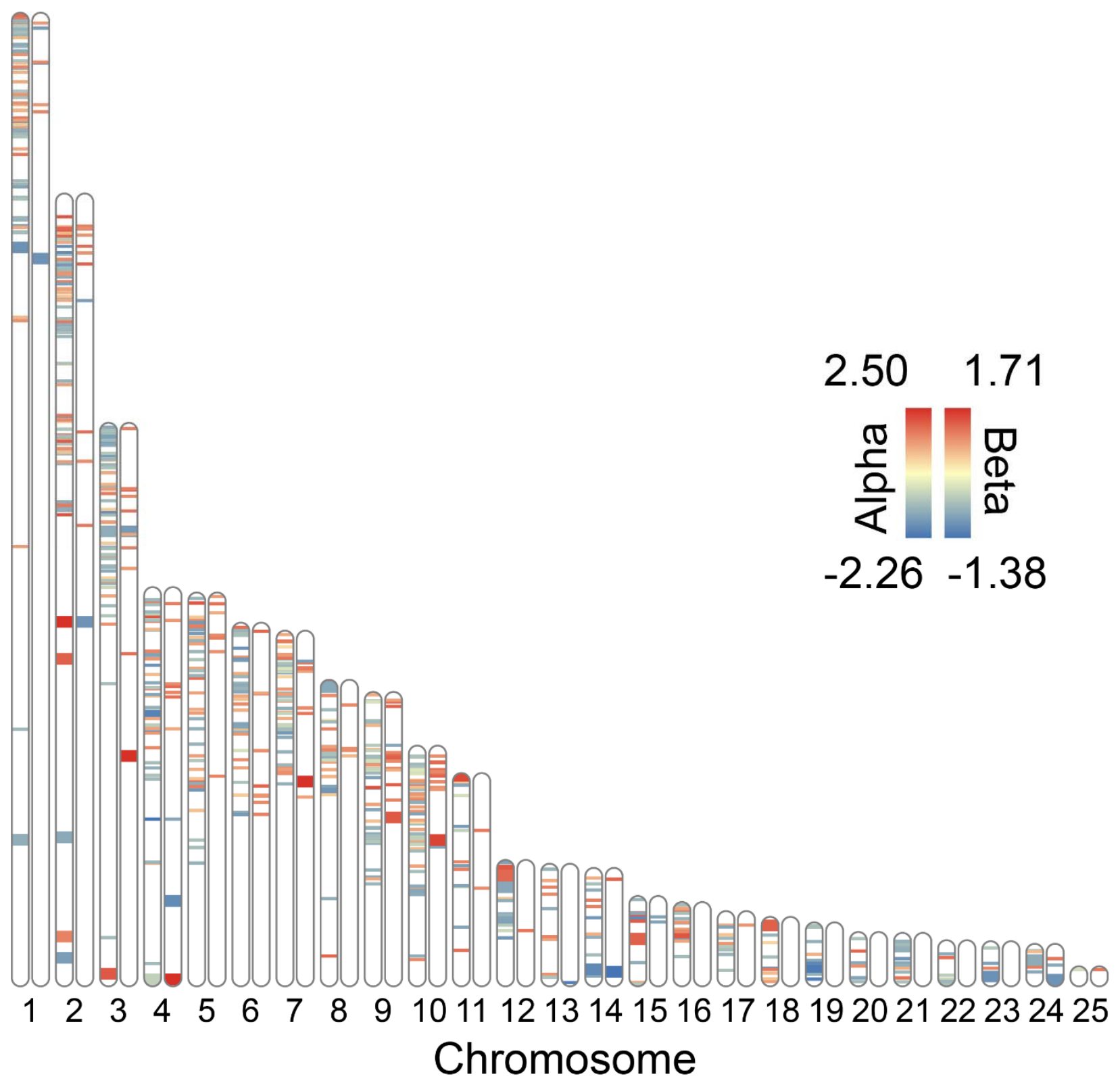

306 Figure 3. Example ideogram plot using Bayesian genomic cline (BGC) outliers for Terrapene

307 ddRAD SNPs (y-axis), plotted onto Trachemys scripta chromosomes (x-axis). Chromosomes are

308 duplicated, with alternative heatmaps for cline center ( $\alpha$; left) and rate ( $\beta$; right). Larger heatmap

309 bands correspond to SNPs located within known genes, whereas smaller bands were found in

310 unknown scaffolds. 


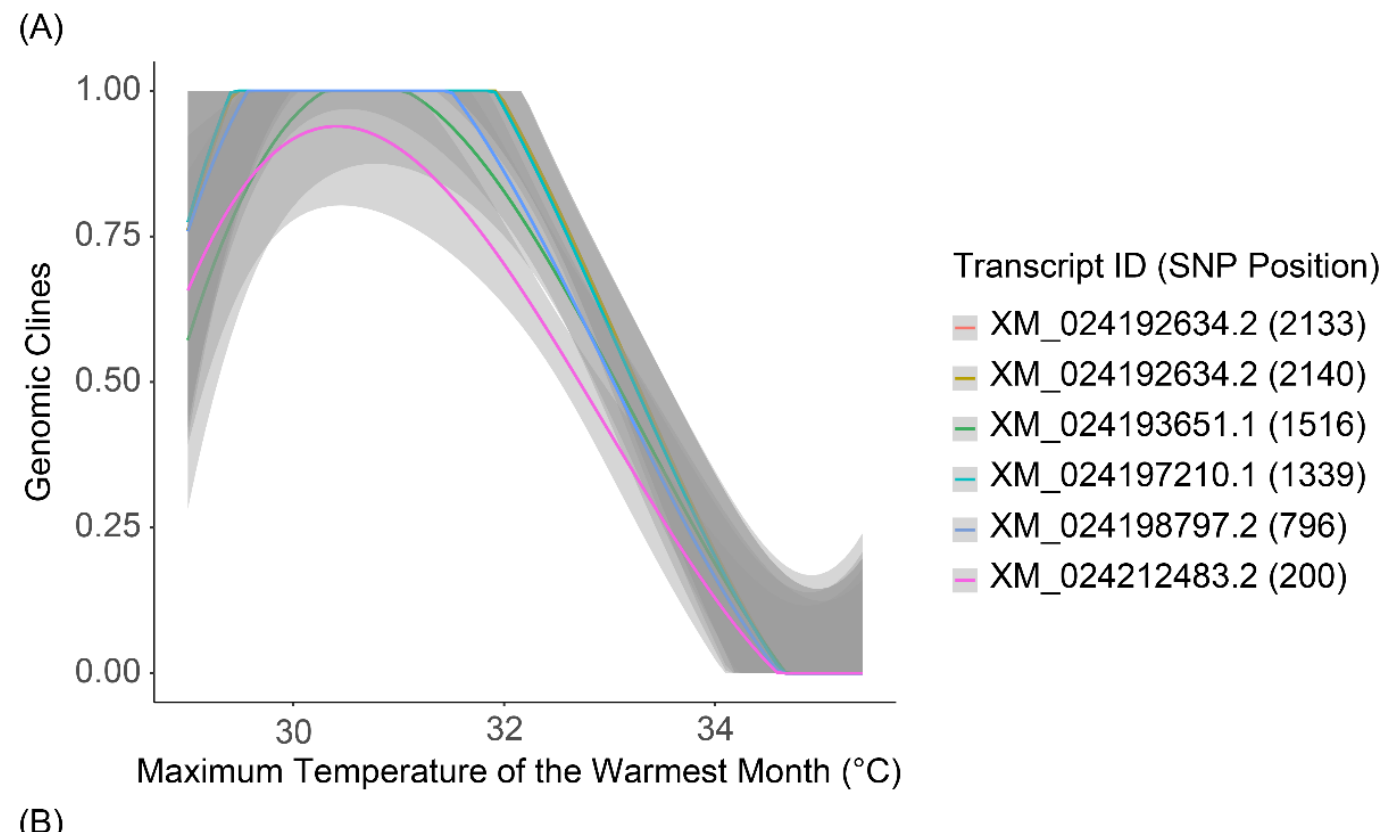

(B)

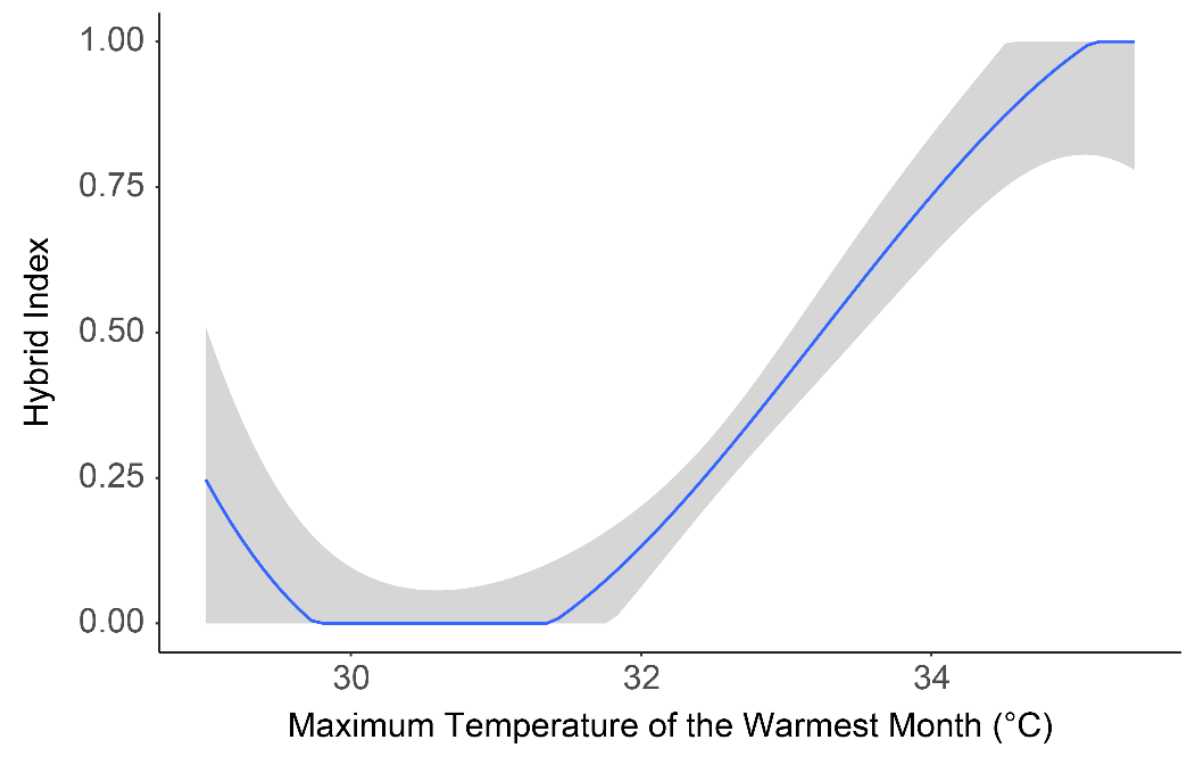

312 Figure 4: Example plots that can be made using the INTROGRESS pipeline in ClinePlOTR. The

313 includes climatic variable on the $\mathrm{X}$-axis corresponds to BioClim raster layer 5

314 (https://worldclim.org). The gray shading indicates confidence intervals for each regression line.

315 (A) Genomic clines for six outlier SNPs mapped to the Terrapene mexicana triunguis

316 transcriptome. Transcript IDs correspond to GenBank accession numbers and the position of

317 each SNP (in base pairs) on the locus. (B) Hybrid index output from INTROGRESS versus an

318 environmental variable. 